\title{
Molecular Dynamics Simulation on Cavitation Bubble Collapse
}

\author{
Chao QIU*, Han CHEN**, Changchun ZHOU*** \\ *Civil Aviation Flight University of China, Guanghan 618300, China, E-mail: chaoqiu1987@163.com \\ **Civil Aviation Flight University of China, Guanghan 618300, China, E-mail: chenghanstorm@sina.com \\ ***Civil Aviation Flight University of China, Guanghan 618300, China, E-mail: zhccj@163.com \\ crossref http://dx.doi.org/10.5755/j01.mech.24.1.15127
}

\section{Introduction}

The cavitation bubbles have been a sustained attention and research since it was discovered in the early twentieth century. Whether it is the propeller of a ship or the dam of a hydropower station, both have suffered serious cavitation damage caused by the collapse of bubbles. Despite negative characterizations of cavitation bubbles are still causing some troubles in life, much more focused attention have been drawn in recent years for its positive application in super cavitation, aerospace, bio-medical [1, 2] and so on.

The spray nozzle is widely used in gas turbine engine, atomization of fuel towards into combustor has a significant influence on engine combustion. The jet flow produced by bubble collapse impact on fuel could make it much more dispersed and form a finely atomized spray. Conversely, in the lubrication system of aero engine, mounts of cavitation bubbles gathered in oil tank would block the flowing of oil and lead a terrible condition for lubrication units. With the development of archaeology around the world, cleaning of antiques is really a headache for archaeologists. The problem could be solved by jet flow caused by bubble collapse, which could impact soil on antiques and remove it [3]. But it is noteworthy that the strength of jet flow should be strictly controlled in order to prevent antiques from being damaged. Similarly, the jet flow of bubble collapse is also an effective method to break down the wall of cancer cell in clinical treatment [4, 5]. In addition, operation of super cavitation weapon is based on the cavitation bubble covering the partly or full surface of submarines [6, 7]. In that case, the rate of submarines could be accelerated significantly for being separated from water by cavitation bubble. However, loud noise caused by bubble collapse is extremely dangerous for submarines. Generally, the dynamic characteristics of cavitation bubble collapse are most important for its application.

In the current research, the dynamic characteristics of cavitation bubble are closed related to viscosity, surface tension and pressure of liquid. Among the factors, the viscosity of liquid plays a dominant role on bubble collapse. It could retard the collapse of cavitation bubble by slowing the rate of bubble contraction [8, 9]. On the contrary, bubble collapse accelerated as the increase of surface tension and pressure [10-12].Furthermore, cavitation bubble collapse varies with the concentration of abrasive particles in fluid [13].

Although the research on dynamics characteristics of bubble collapse has made a remarkable progress, some details are still interrogative due to the limitation of experimental technology. Molecular dynamics simulation (MD) is a method of micro/nano scale, which could simulate bubble collapse according to the trajectory of molecules and even reveal the characteristics of bubble collapse. This method has been used by Moore and Tsuda to simulate the nucleation of cavitation bubble $[14,15]$. However, bubble collapse is not involved in their researches, which is extremely important for its application. This word, molecular dynamics simulation is used to simulate the collapse of cavitation bubble, and the effect of temperature on bubble collapse is both considered to reveal the dynamic characteristics of bubble collapse.

\section{Mathematical model and numerical methods}

Argon atom is usually used in molecular dynamic simulation for it's a monatomic molecule. A mathematical model is proposed and initial distribution of atoms is shown in Fig. 1. 2680 liquid argon atoms surrounding a cavitation bubble are distributed in a three-dimensional computational domain, with diameter of bubble is $d=4.64 \sigma$. Gas argon atoms composed of cavitation bubble are negligible, who have little impact on liquid argon atoms as the number of gas atoms are much less than liquid atoms. The canonical ensemble is used, in which a temperature coefficient is introduced to keep the temperature constant. And also the periodic boundary conditions are set to ensure the number of atoms remain the same. It means one atom moves out of the computational domain, there must be another atom moves in from opposite boundary with the same velocity and acceleration.

In general, nomenclatures are usually used in molecular dynamic simulation for extremely small of molecular dynamic parameters. They are list in Table 1.

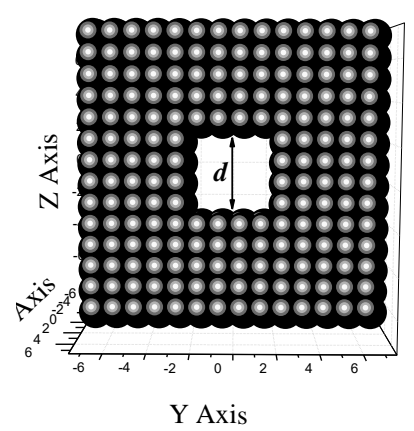

Fig. 1 Initial distribution of atoms 
Table 1

Relationship between nomenclatures and MD parameters

\begin{tabular}{|c|c|c|c|}
\hline MD parameters & SI & Nomenclatures & Relationship \\
\hline Simulation domain size $\quad L$ & $\mathrm{~m}$ & $L^{*}$ & $L / \sigma$ \\
\hline Boltzmann constant $k_{B}$ & $\mathrm{~J} / \mathrm{K}$ & & \\
\hline Mass of atom $m$ & $\mathrm{~kg}$ & & \\
\hline Pressure $P$ & $\mathrm{~Pa}$ & $P^{*}$ & $P \sigma^{3} / \varepsilon$ \\
\hline Distance between atoms $i$ and $j \quad r_{i j}$ & $\mathrm{~m}$ & $r_{i j}{ }^{*}$ & $r_{i j} / \sigma$ \\
\hline Cut-off radius $r_{c}$ & $\mathrm{~m}$ & $r_{c}{ }^{*}$ & $r_{c} / \sigma$ \\
\hline Simulation time $t$ & $\mathrm{~s}$ & $t^{*}$ & $\left(\varepsilon / m \sigma^{2}\right)^{1 / 2} t$ \\
\hline System temperature $T$ & $\mathrm{~K}$ & $T^{*}$ & $k_{B} T / \varepsilon$ \\
\hline Scale of energy for potential functions $\varepsilon$ & $\mathrm{kcal} / \mathrm{mol}$ & & \\
\hline Scale of length for potential functions $\sigma$ & $\mathrm{m}$ & & \\
\hline Potential energy $U$ & $\mathrm{~J}$ & $U^{*}$ & $U / \varepsilon$ \\
\hline Total energy $E$ & $\mathrm{~J}$ & $E^{*}$ & $E / \varepsilon$ \\
\hline Velocity of atom $i \quad v_{i}$ & $\mathrm{~m} / \mathrm{s}$ & & \\
\hline Force impact on atom $i \quad F_{i}$ & $\mathrm{~N}$ & $F_{i}^{*}$ & $F_{i} \sigma / \varepsilon$ \\
\hline Displacement of atom $i \quad S_{i}$ & $\mathrm{~m}$ & $S_{i}^{*}$ & $S_{i} / \sigma$ \\
\hline Diameter of simulation bubble $d$ & $\mathrm{~m}$ & $d^{*}$ & $d / \sigma$ \\
\hline
\end{tabular}

The intermolecular interactions in MD are described by the well-known Lennard-Jones potential function (Eq. 1):

$$
\begin{aligned}
& U\left(r_{i j}\right)=4 \varepsilon\left[\left(\frac{\sigma}{r_{i j}}\right)^{12}-\left(\frac{\sigma}{r_{i j}}\right)^{6}\right], r_{i j}<r_{c}, \\
& U\left(r_{i j}\right)=0, r_{i j} \geq r_{c},
\end{aligned}
$$

where $\sigma$ and $\varepsilon$ are the scales of length and energy for potential function, respectively. For argon atom, $\varepsilon=$ $=0.24 \mathrm{kcal} / \mathrm{mol}, \sigma=3.405 \AA$. $U$ is potential energy of atoms, and $r_{i j}$ is distance between atom $i$ and atom $j$. In this MD study, the cut-off radius $r_{c}$ is set at $2.5 \sigma$, beyond which the pair interaction is neglected. Furthermore, time step $t^{*}=4.5 \times 10^{-5}$ (almost $1 \mathrm{fs}$ ).

Position of atom $i$ at any time could be calculated based on the Newton's laws of motion:

$$
\overrightarrow{S_{i}}\left(t+\frac{1}{2} \delta t\right)=\overrightarrow{S_{i}}\left(t-\frac{1}{2} \delta t\right)+\overrightarrow{v_{i}}\left(t+\frac{1}{2} \delta t\right) \delta t+\frac{1}{2} \frac{\overrightarrow{F_{i}}(t)}{m} \delta t^{2}
$$

where, $m$ is mass of atom, and $m=6.63 \times 10^{-26} \mathrm{~kg}$. $F_{i}(t)$ is the force impact on atom $i$ :

$$
\overrightarrow{F_{i}}(t)=\frac{d U}{d r}
$$

and velocity of atom $i$ could be described as:

$$
\overrightarrow{v_{i}}\left(t+\frac{1}{2} \delta t\right)=\overrightarrow{v_{i}}\left(t-\frac{1}{2} \delta t\right) \xi+\frac{\overrightarrow{F_{i}}(t)}{m} \delta t
$$

$$
\xi^{2}=\frac{\left[3(N-1) k_{B} T_{s e t} / m\right]}{\sum_{i=1}^{N}\left[\overrightarrow{v_{i}}\left(t-\frac{1}{2} \delta t\right)\right]^{2}},
$$

where $T_{\text {set }}$ is rated temperature, and $k_{B}$ is Boltzmann constant.

Combining Eqs. (1) - (5) [16], movement of atoms as well as potential energy could be obtained. And bubble collapse process could be analyzed as well. This simulation is carried on the IBM system X3800 computer, and programmed by ourselves in Fortran language.

Molecular dynamics simulation of bubble collapse lasts almost 440 ps (including 440, 000 time steps). Simulation temperature oscillated at the beginning of simulation (about $40 \mathrm{ps}$ ), and then thermal equilibrium is reached. After that, simulation data including the potential energy, total energy and position of atoms are recorded to analyze the dynamic characteristics of bubble collapse.

\section{Results and discussion}

\subsection{Bubble collapse process}

Fig. 2 is the collapse process of cavitation bubble with $T^{*}=0.62$.

After reaching thermal equilibrium, the cavitation bubble is gradually compressed until it disappeared in liquid. Actually, the bubble does not really disappeared but breaking up into a lot of nucleus and dissolving in liquid.

Additionally, bubble collapse process could also be analyzed in term of potential energy. Fig. 3 is potential energy curve, which describes the potential energy varying with the distance between atoms. According to Lennard-Jones potential function, potential energy is maximum at $r=1.122 \sigma$, while the initial distance between atoms in this work is $r_{0}=1.161 \sigma$.

where, $\xi$ is temperature coefficient: 


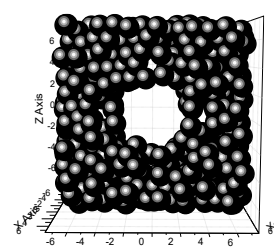

$40 \mathrm{ps}$

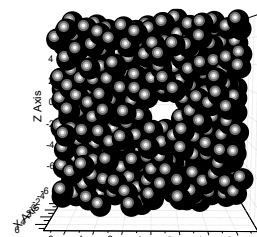

$160 \mathrm{ps}$

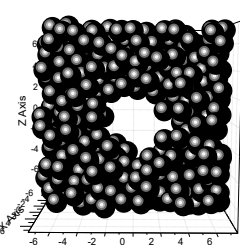

$80 \mathrm{ps}$

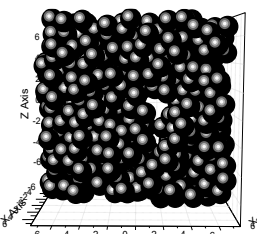

$200 \mathrm{ps}$

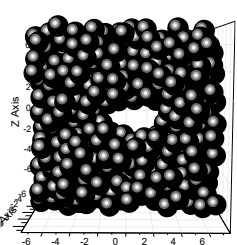

$120 \mathrm{ps}$

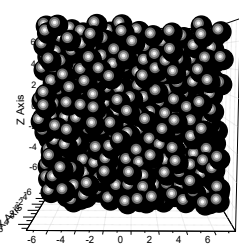

$240 \mathrm{ps}$
Fig. 2 Collapse process of cavitation bubble with $T^{*}=0.62$

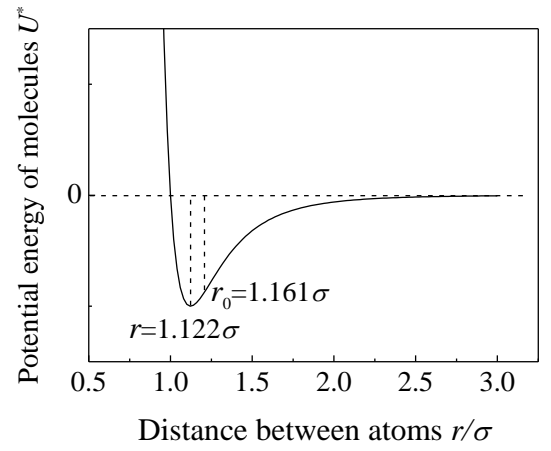

Fig. 3 Potential energy varying with distance between atoms

Variation of potential energy during bubble collapse is shown in Fig. 4, which decreases gradually in $t_{c}$, and then keeps constant until the end of simulation.

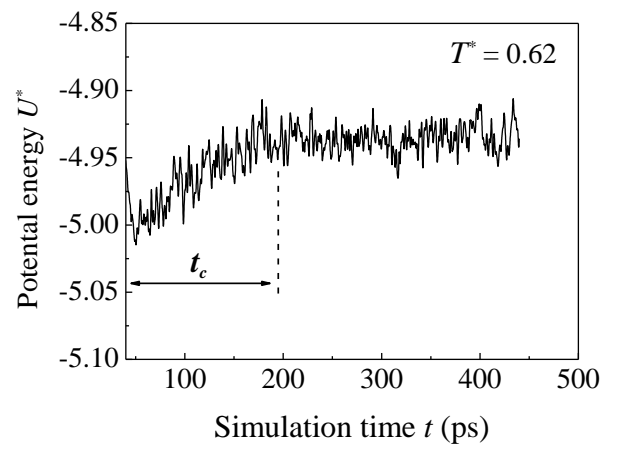

Fig. 4 Variation of potential energy during bubble collapse

According with potential energy curve and the $r$ in Fig. 3, potential energy could only decrease when distance between atoms increasing. However, both the shape and size of computational domain are unchangeable in canonical ensemble, the space of liquid must be expansive as the increase of distance between atoms. In that case, cavitation bubble has to be compressed for the expansion of liquid and finally breaking up into a lot of nucleus in liquid. Therefore, $t_{c}$ is collapse time of bubble. Flowing that, potential energy changes little and nothing will happen in computational domain.

\subsection{Effect of temperature on cavitation bubble collapse}

Temperature has a significant effect on bubble

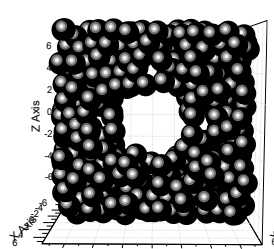

$40 \mathrm{ps}$

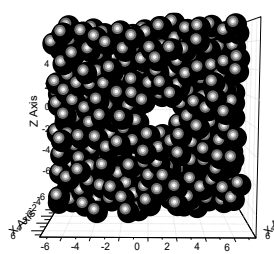

$160 \mathrm{ps}$

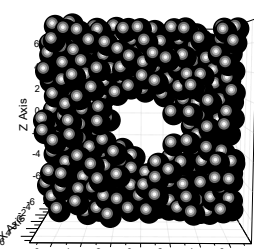

$80 \mathrm{ps}$

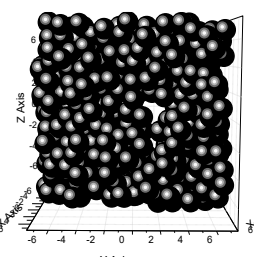

200 ps

a

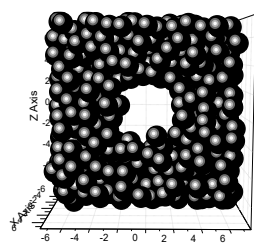

$40 \mathrm{ps}$

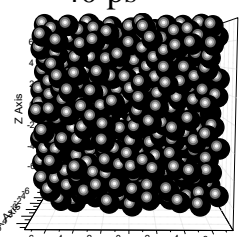

160 ps

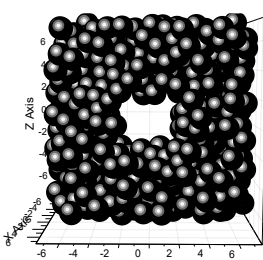

$40 \mathrm{ps}$

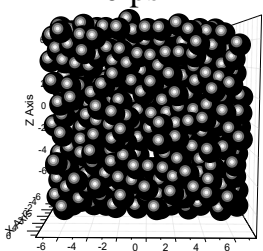

160 ps

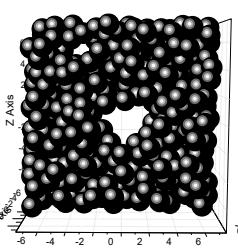

80 ps

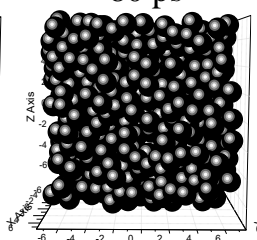

$200 \mathrm{ps}$

b

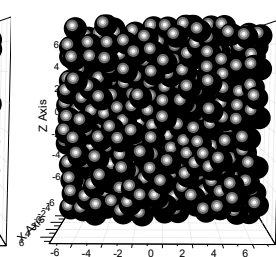

$80 \mathrm{ps}$

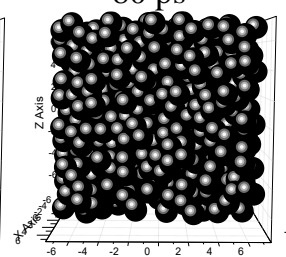

$200 \mathrm{ps}$

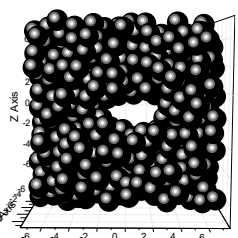

$120 \mathrm{ps}$

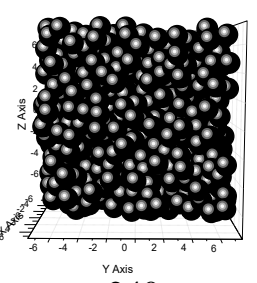

240 ps

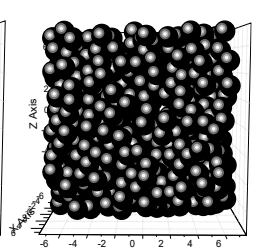

$120 \mathrm{ps}$

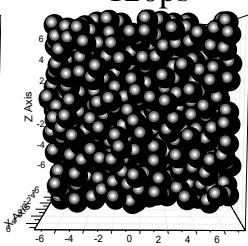

240 ps

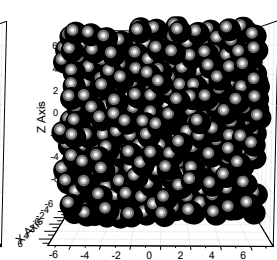

$120 \mathrm{ps}$

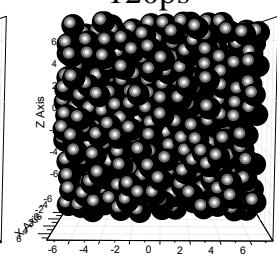

240 ps
Fig. 5 Bubble collapse in different temperatures: $\left(\mathrm{a}: T^{*}=\right.$ $\left.0.62 ; \mathrm{b}: T^{*}=0.70 ; \mathrm{c}: T^{*}=0.80\right)$

collapse. Fig.5 shows the collapse process of bubble in different temperature, which are $T^{*}=0.62, T^{*}=0.70$ and $T^{*}=0.80$ respectively.

It is obvious that bubble collapse faster as the increase of temperature. Also it could be proved by potential energy in Fig. 6. Variation of potential energy is almost the same with different temperature, all of which reducing at first, but $t_{c}$ becomes shorter with the increase of temperature. Namely, bubble collapse is accelerated when temperature rising, which according with the behaviors of bubble in Fig.5. 


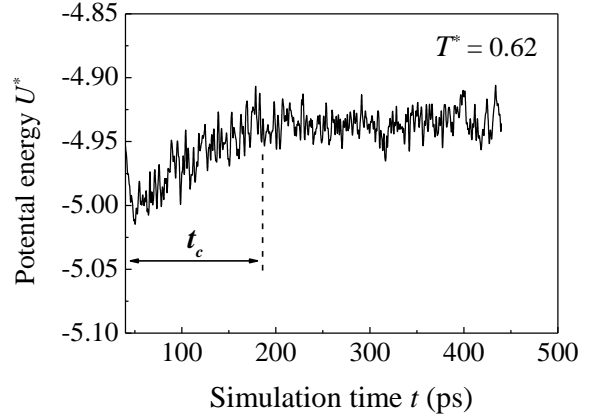

a

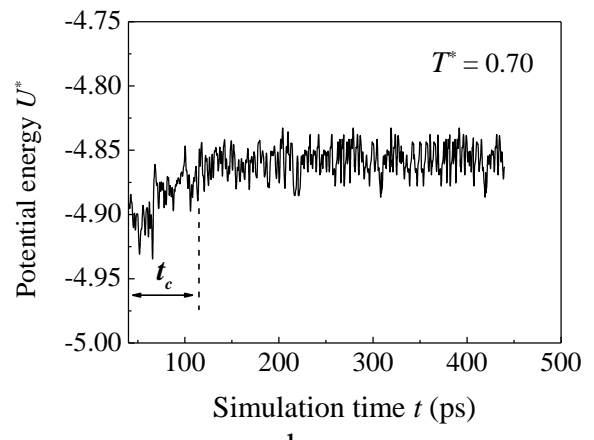

b

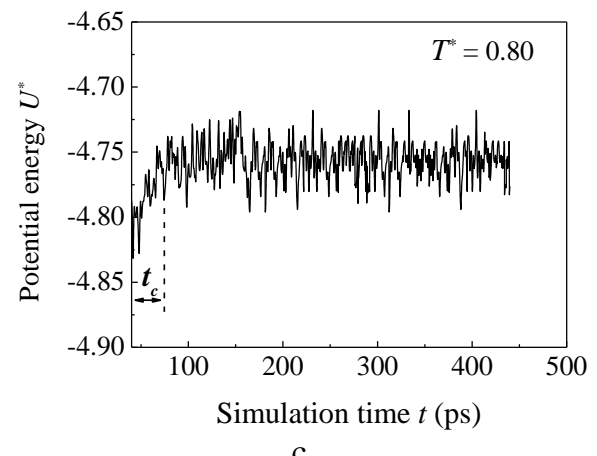

Fig. 6 Variation of potential energy in different temperature:

(a: $\left.T^{*}=0.62 ; \mathrm{b}: T^{*}=0.70 ; \mathrm{c}: T^{*}=0.80\right)$

A more detailed analysis of collapse time $t_{c}$ in different temperatures is presented in Fig. 7.

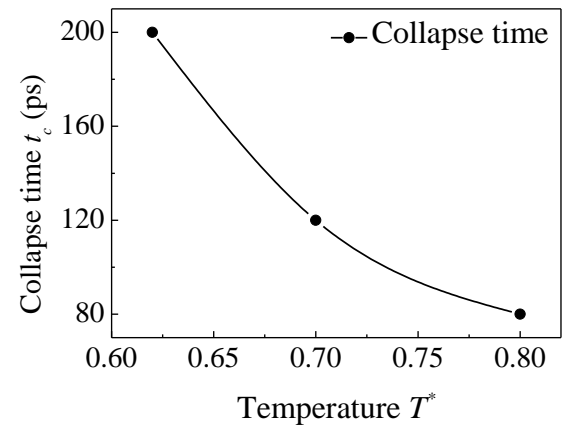

Fig. 7 Collapse time varying with temperature

It could be known that collapse time is almost shortened by $60 \%$ with temperature increasing from $T^{*}=$ 0.62 to $T^{*}=0.80$, but acceleration of bubble collapse slows down. This is primarily linked to the viscosity of liquid. It is well known viscosity could retard the collapse of cavitation bubble. However, viscosity decreases gradually with the increasing temperature, and the influences of liquid viscosity on bubble collapse has weakened the same time, which resulting in bubble collapsing faster.

\subsection{Effect of temperature on collapse strength of bubble}

Temperature also affects the collapse strength of cavitation bubble. Total energy of bubble collapse in different temperatures is shown in Fig. 8.
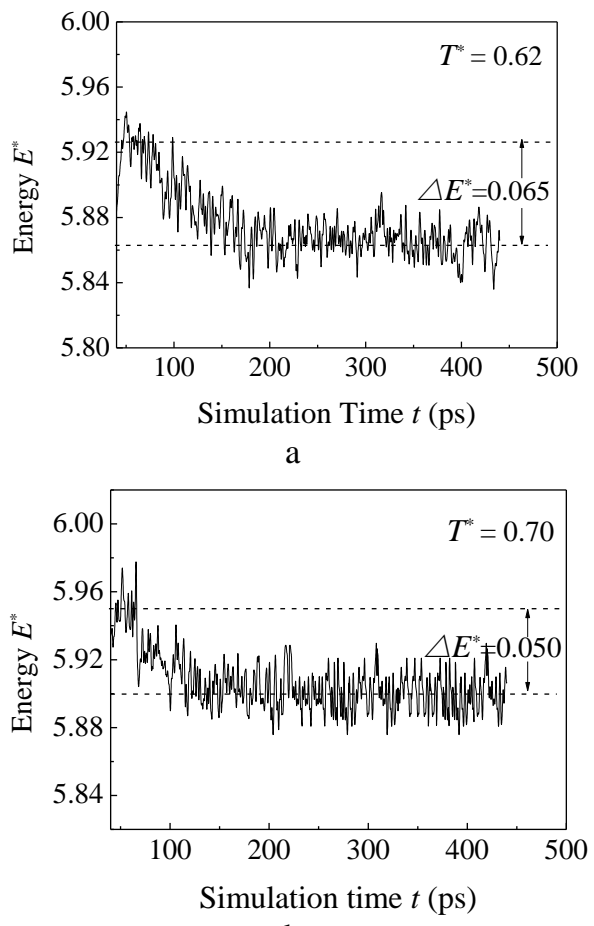

b

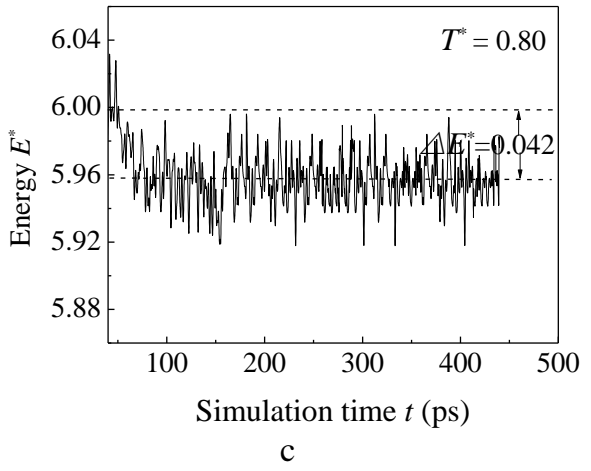

Fig. 8 Variation of total energy in different temperature: (a: $\left.T^{*}=0.62 ; \mathrm{b}: T^{*}=0.70 ; \mathrm{c}: T^{*}=0.80\right)$

In Fig. 8, total energy decreases gradually during bubble collapse, and $\Delta E^{*}$ is the energy released by bubble collapsing. It could be found the energy released by bubble collapsing becomes smaller with the increase of temperature, Namely, jet flow caused by bubble collapse is weaker when temperature rising. Strength of jet flow is almost reduced by $35 \%$ with temperature increasing from $T^{*}=$ $=0.62$ to $T^{*}=0.80$. This is primarily linked to the surface tension of liquid. Surface tension decreases gradually with the increasing temperature, and strength of jet flow reduce the same time.

In general, temperature should be reasonable for cavitation bubble application. For example, solution with higher temperature is more suitable for cleaning antiques. Rising the temperature could improve the efficiency of removing soil on antiques without any damage. On the contrary, super cavitation weapons sails faster in cooler fluid for bubble existing more time on submarine surface. 


\section{Conclusion}

Temperature is significant for dynamic characteristics of bubble collapse. It concludes that bubble collapses faster as the increase of temperature, but the jet flow becomes weaker the same time. In addition, collapse time of cavitation bubble is almost shortened by $60 \%$, and the strength of jet flow could be reduced by $35 \%$ with temperature varies from 0.62 to 0.80 .

\section{Acknowledgment}

This research was supported by National Natural Science Foundation of China(No.11602293), Science and Innovation Guidance Foundation of CAAC(No.0251520), Innovation Team of Civil Aero-engine Airworthiness and Maintenance(No.JG2016-33).

\section{References}

1. Shimizu, Y.; Takashima, A.; Satoh, W.; et al. 2009. Biochip with integrated pumps for plug-based sequential exchange of solutions, Sensors \& Actuators B Chemical 140(2): 649-655. http://dx.doi.org/10.1016/j.snb.2009.04.048.

2. Volokh, K.Y. 2015. Thrombus rupture via cavitation, Journal of Biomechanics 48(10): 2186-2188. http://dx.doi.org/10.1016/j.jbiomech.2015.04.044.

3. Niemczewski, B. 2014. Cavitation intensity of water under practical ultrasonic cleaning conditions, Ultrasonics Sonochemistry 21(1): 354-359. http://dx.doi.org/10.1016/j.ultsonch.2013.07.003.

4. Li, X. 2009. Lyp-1 ultrasonic microbubbles targeting to cancer cell as tumor bio-acoustics markers or drug carriers: targeting efficiency evaluation in microfluidic channels, $31^{\text {st }}$ Annual International Conference of the IEEE Engineering in Medicine and Biology Society, 2009. Hilton Minneapolis, Minnesota, USA463-466.

5. Liu, Q.; Dai, L. 2006. Research progresses about the mechanism of ultrasonic cavitation and its applications in anticancer domain, Journal of Ultrasound in Clinical Medicine 8(4): 235-238.

6. Vanek, B.; Bokor, J.; Balas, G.J.; et al. 2006. Longitudinal motion control of a high-speed supercavitation vehicle, Journal of Vibration \& Control 13(2): 159-184. http://dx.doi.org/10.1177/1077546307070226.

7. Shang, Z. 2013. Numerical investigations of supercavitation around blunt bodies of submarine shape, Applied Mathematical Modelling 37: 8836-8845. http://dx.doi.org/10.1016/j.apm.2013.04.009.

8. Aghdam, A.H.; Ohl, S. W.; Khoo, B.C.; et al. 2012. Effect of the viscosity on the behavior of a single bubble near a membrane, International Journal of Multiphase Flow 47(3): 17-24. https://doi.org/10.1016/j.ijmultiphaseflow.2012.06.010.

9. Lu, M.; Ning, Z.; Yan, K.; et al. 2014. Breakup of cavitation bubbles within the diesel droplet, Chinese Journal of Mechanical Engineering 27(1): 198-204. https://doi.org/10.3901/CJME.2014.01.198.

10. Ehsan, S.; Mehrzad, S.; Reza, E. 2011. A novel numerical scheme for the investigation of surface tension effects on growth and collapse stages of cavitation bubbles, European Journal of Mechanics B/Fluids 30: 41-50. https://doi.org/10.1016/j.euromechflu.2010.09.002.

11. Brouwer, G. C.; Wagner, E.C.; Van Ommen, J.R.; et al. 2012. Effects of pressure and fines content on bubble diameter in a fluidized bed studied using fast X-ray tomography, Chemical Engineering Journal 207-208: 711-717. https://doi.org/10.1016/j.cej.2012.07.040.

12.Li, B.B.; Zhang, H.C.; Lu, J.; et al. 2011. Experimental investigation of the effect of ambient pressure on laser-induced bubble dynamics, Optics \& Laser Technology 43: 1499-1503.

https://doi.org/10.1016/j.optlastec.2011.05.016.

13. Boudreau, B.P. 2012. The physics of bubbles in surficial, soft, cohesive sediments, Marine and Petroleum Geology 38: 1-18.

http://dx.doi.org/10.1016/j.marpetgeo.2012.07.002.

14. Moore, E.; Corrales, L.R.; Desai, T.; et al. 2011. Molecular dynamics simulation of Xe bubble nucleation in nanocrystalline UO2 nuclear fuel, Journal of Nuclear Materials 419(1): 140-144.

http://dx.doi.org/10.1016/j.jnucmat.2011.08.052.

15. Tsuda, S.I.; Shu, T.; Matsumoto, Y. 2008. A study on the growth of cavitation bubble nuclei using large-scale molecular dynamics simulations, Fluid Dynamics Research 40: 606-615. http://dx.doi.org/10.1016/j.fluiddyn.2008.02.002.

16. Park, S.H.; Weng, J.G.; Tien, C.L. 2001. A molecular dynamics study on surface tension of microbubbles, International Journal of Heat and Mass Transfer 44: 1849-1856.

http://dx.doi.org/10.1016/S0017-9310(00)00244-1.

\section{Chao Qiu, Han Chen, Changchun Zhou}

\section{MOLECULAR DYNAMICS SIMULATION ON CAVITATION BUBBLE COLLAPSE}

S u m m a r y

The characteristics of bubble collapse are especially important for its application in cavitation-protection, super cavitation and aerospace. In that case, it is necessary to control the collapse process of bubble accurately according to requirements. In this paper, molecular dynamics simulation is used to study the dynamic characteristics of single bubble in canonical ensemble condition. Collapse process of bubble is described by analyzing the potential energy and movement of molecules. Effects of temperature on bubble dynamics characteristics are discussed especially. The result indicates that bubble collapses faster as the increase of temperature, but the strength of jet flow caused by collapsing becomes weaker the same time. It finds that the collapse time of bubble is almost shortened by $60 \%$, and the strength of jet flow could be reduced by $35 \%$ with temperature varies from 0.62 to 0.80 .

Keywords: Cavitation bubble, Temperature, Collapse time, Strength of jet flow.

Received June 03, 2016 Accepted February 15, 2018 